\title{
Pericardial effusion reveals an hepatopericardial fistulas of hepatic echinococcosis (A case report)
}

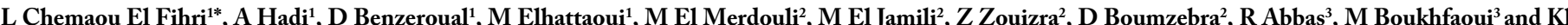
Rabbani $^{3}$

${ }^{1}$ Department of Cardiology, University Hospital Centre Mohammed VI of Marrakech

${ }^{2}$ Department of Cardiovascular surgery, University Hospital Centre Mohammed VI of Marrakech

${ }^{3}$ Department of visceral surgery, University Hospital Centre Mohammed VI of Marrakech

\begin{abstract}
Hydatidosis is a parasitic infestation which is endemic to sheepherding regions of the world. Hydatid cysts are most commonly found in the liver and lungs, but they are rarely found in pericardium. In our case, the cyst was not a primary cardiac hydatid cyst. In this article, we report a 62 -year-old male case of pericardial effusion due to fistulisation of a hepatic hydatic cyst to the pericardium. The patient was managed chirurgically, followed by medical treatment. In regions where hydatid disease is endemic, the diagnosis of hydatid cyst should always be considered in the differential diagnosis.
\end{abstract}

\section{Introduction}

Hydatid disease (HD) is a zoonotic disorder caused by parasites that belong to the Echinococcus species of the Taeniidae family of cestodes. The Echinococcus species that most commonly causes HD in humans are Echinococcus granulosus. Hydatid disease can affect any organ and may involve any anatomical site of the human body. However, even in countries where hydatid disease is endemic, only sporadic isolated cases of cardiac involvement $(0.5-2 \%$ of all cases) have been reported [1]. Hepatic hydatid cysts are usually asymptomatic and may be detected incidentally by imaging. Hepatic hydatid cysts may be symptomatic due to mass effect or because of complications such as rupture or infection.

In this study, we will elaborate the case of an infected hepatic hydatid cyst fistulized in the pericardium and revealed by a pericardial effusion.

\section{Observation}

A 62-year-old man, without particular pathological antecedents, especially no notion of contact with dogs, who has had precordial chest pain for five days, associated with dyspnea classe III of New York Heart Association (NYHA), all evolving in a context of deterioration of the general state. On clinical examination, the patient is in reasonably good general condition, his blood pressure is $115 / 68 \mathrm{~mm} \mathrm{Hg}$, his pulse is 100 beats per minute and his breathing rate is 19 cycles per minute. Cardiovascular examination shows deafening of heart sounds without signs of right heart failure. Abdominal examination shows epigastric and right hypochondrium sensibility. Besides, the ECG shows a diffuse ST segment elevation without mirror image with a sub-shift of PQ (Figure 1), and the chest radiography shows cardiomegaly with uprising of the right diaphragmatic dome (Figure 2).

The transthoracic echocardiograph confirmed the presence of pericardial effusion with moderate abundance without signs of a tamponade (Figure 3).The abdominal ultrasonography individualizes in the liver a well-defined oval formation with a finely echogenic heterogeneous content measuring $10.6 \times 10.2 \mathrm{~cm}$ evoking an infected hydatid cyst. A complement with chest $\mathrm{CT}$ has objected a liver collection probably related to an infected hydatid cyst responsible of a pericardial reaction effusion (Figure 4). The biological assessment shows a white blood cell count of $13740 / \mathrm{UL}$ with predominantly eosinophils, a CRP of $300 \mathrm{mg} / \mathrm{L}$ and positive hydatid serology. Faced with aggravation of the symptomatology by the appearance of a tamponnade, an evacuating puncture before the surgical procedure was carried out urgently and allowed to remove a purulent greenish-colored liquid (Figure 5A). The surgical procedure consists in a costal cystectomy with highlighting during the gesture of a cysto-pericardial fistula individualizable by opacification after sternotomy. Examination of pericardial fluid shows cytology high cellularity made of neutrophils with a positive culture made of gram positive Cocci A chain. Parasitological examination is negative. The anatomopathological examination of the membranes confirms the diagnosis of hydatid cyst. Antibiotherapy was then applied using albendazole. The patient recovered well and was discharged on the postoperative (Figure 5B and 5C).

\section{Discussion}

Hydatid disease is a zoonotic parasitic disease caused by the Echinococcus tapeworm. Echinococcus granulosus and Echinococcus multilocularis are the main causative infectious organisms. E. granulosus is the most common cause of hydatid disease in humans, and it can be found in almost any part of the world. Dogs are the most common primary host, and sheep are the intermediate vector. Humans may be affected after ingestion of food contaminated with the parasite's eggs. Hydatid disease is prevalent in rural sheep-raising areas where

*Correspondence to: L Chemaou El Fihri, Department of Cardiology, University Hospital Centre Mohammed VI of Marrakech, Morocco, E-Mail: laila.chemaou@gmail.com

Key words: fistula, hydatid disease, pericardium

Received: May 22, 2019; Accepted: June 21, 2019; Published: June 26, 2019 


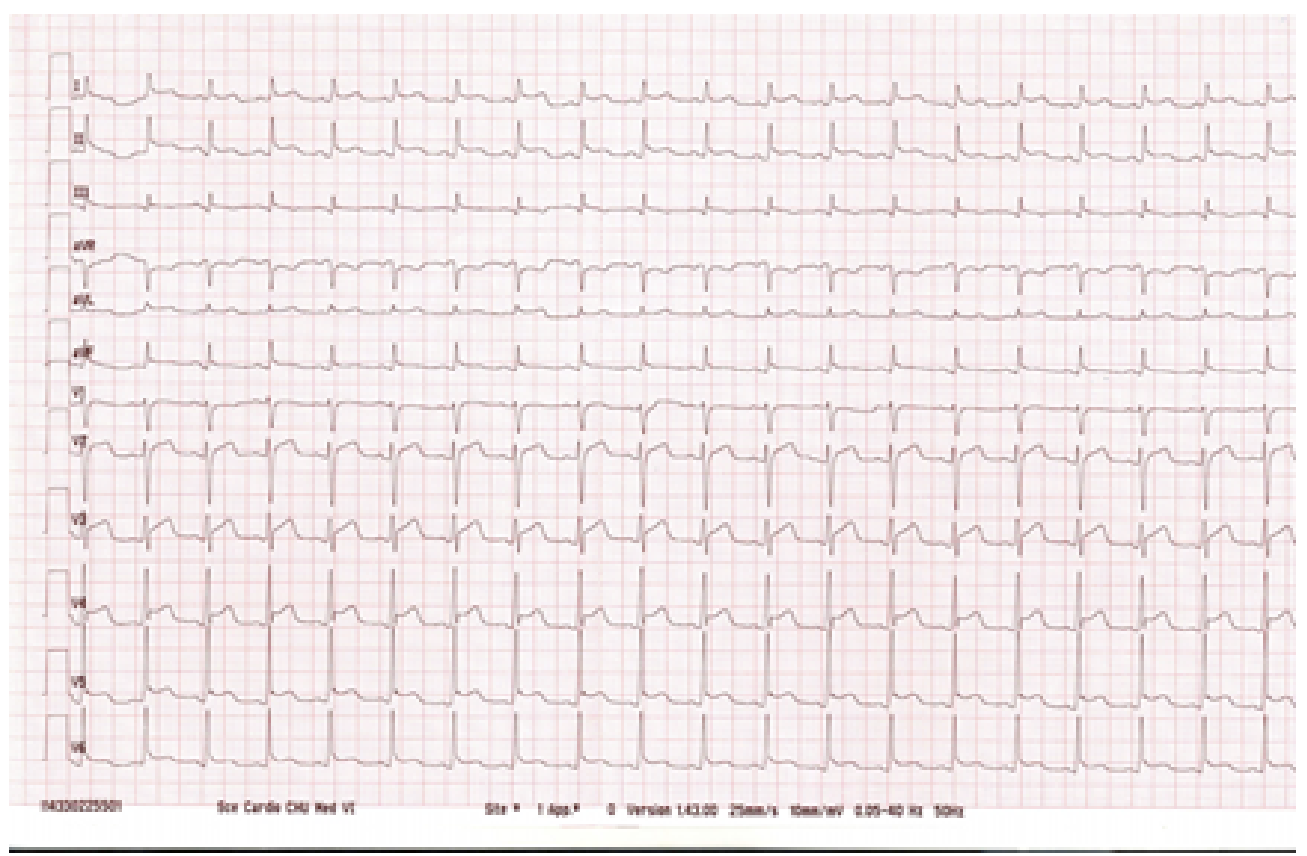

Figure 1. Patient's electrocardiogram at the admission

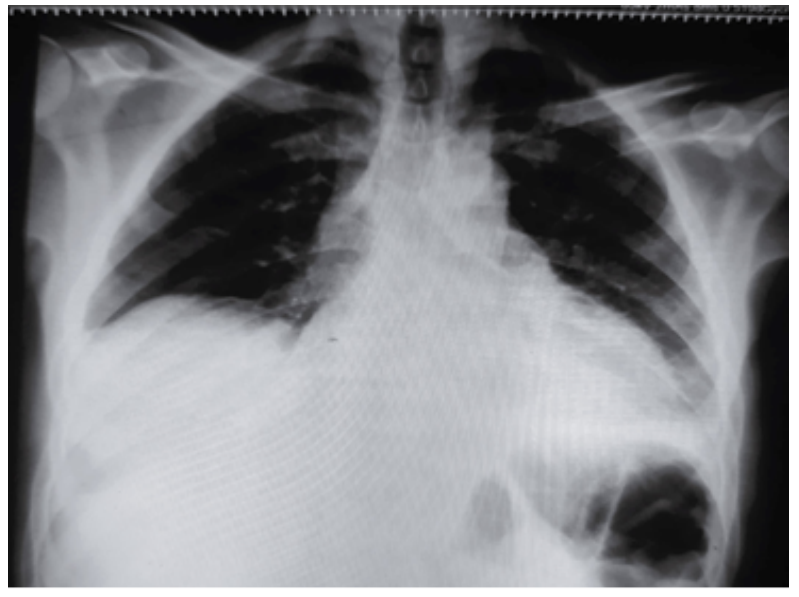

Figure 2. Chest radiography showing cardiomégalie with uprising of the right diaphragmatic dome

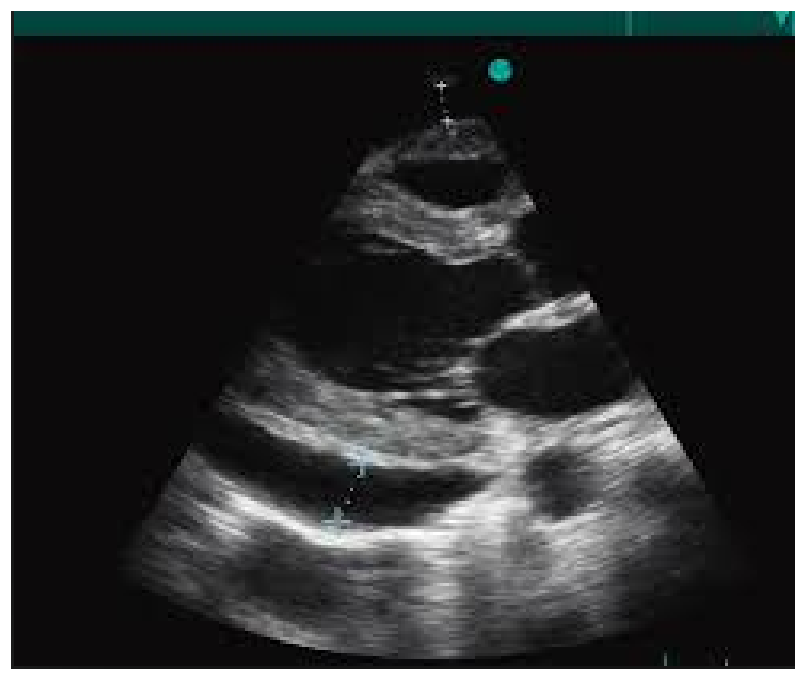

Figure 3. Apical cut large axis showing the pericardial effusion 

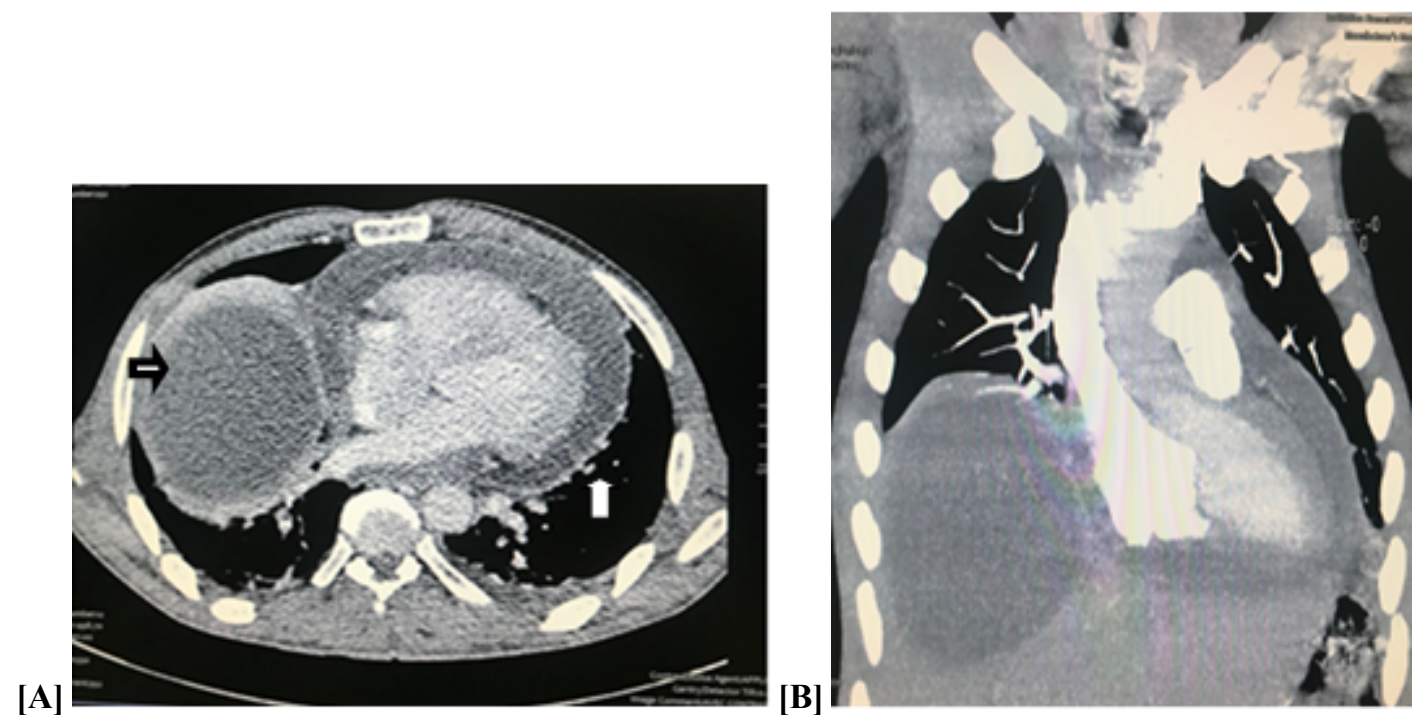

Figure 4. Computed tomography images $(\mathrm{A}, \mathrm{B})$ showing the thickened pericardium with pericardial effusion (white arrow) and the regularly contoured lesion in the hepatic dome (black arrow)

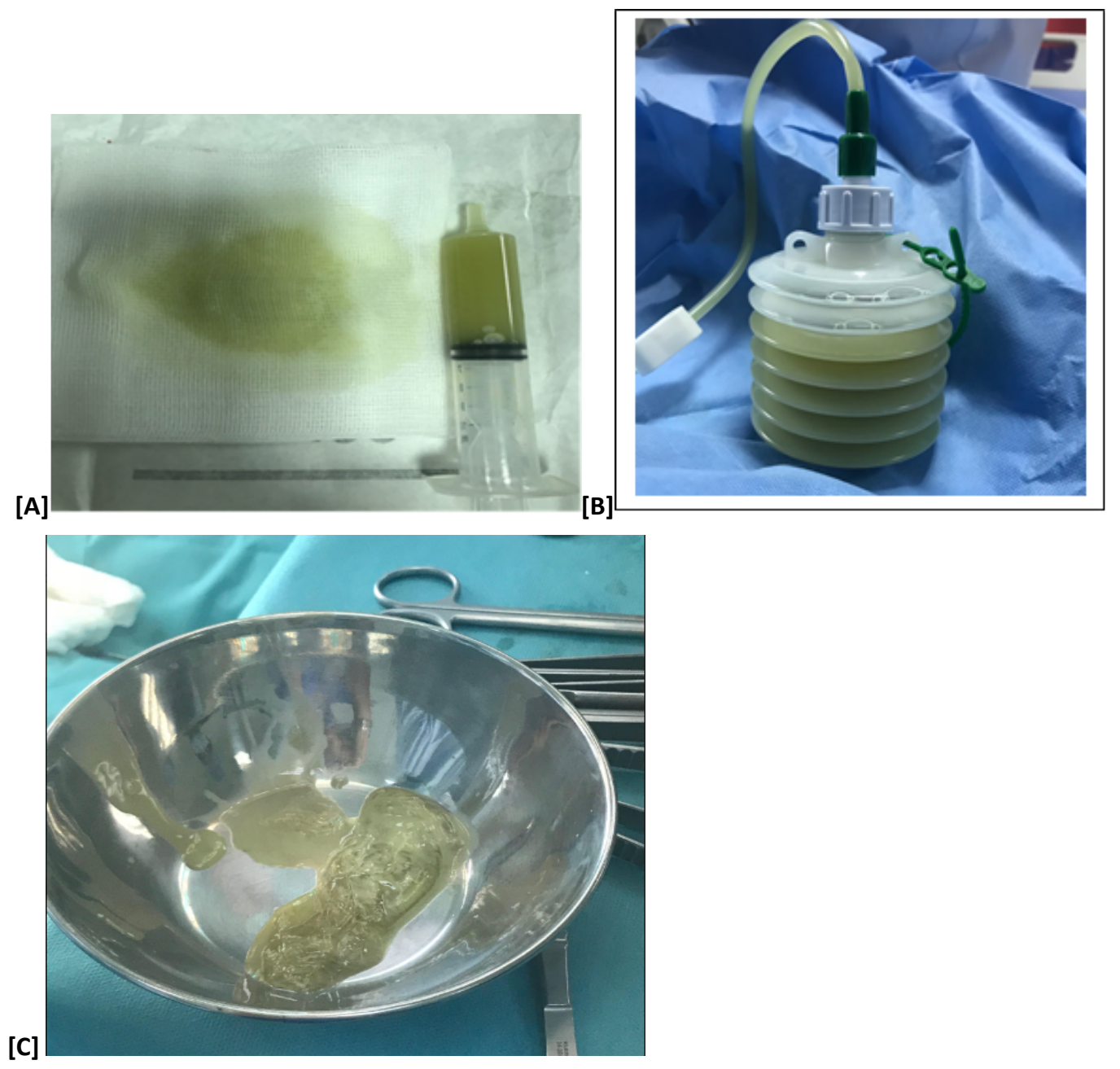

Figure 5. $[A]$ and $[B]$ Images showing the greenesh liquid of the puncture $[\mathrm{C}]$ image of the hydatid membrane during the surgery 
people have a closer contact with dogs and other carnivorous animals. Regions of high prevalence include the Middle East, the Mediterranean, South America, Africa, Australia, and New Zealand [2,3].

The liver is the most likely organ to be involved (55-70\%) through portal drainage, but any organ may be infected. The helminth usually reaches the heart via coronary circulation. The primary hydatid cyst of the heart is rare and has a marked tendency to rupture either into the lumen of a cardiac chamber or into the pericardial sac, depending on its primary location and on the direction of least resistance [4]. However, in our case the cyst was not a primary cardiac hydatid cyst. The hydatid fluid of the hepatic hydatid cyst reached the pericardium through a fistula's tract, leading to pericardial effusion. In a similar case, the cyst invaded the diaphragm and extended to the posterior mediastinum, compressing the pericardium and oesophagus. Moreover, it produced a secondary Budd-Chiari syndrome by occluding the suprahepatic left vein near its outfall in the vena cava and near the inferior vena cava [5].

As HD has a slow growth rate, a significant proportion of cases may remain asymptomatic for years. Hence, asymptomatic cases are incidentally diagnosed by radiological studies performed for other indications [6,7]. However, a minority of cases may show some signs and symptoms depending on the size, number, and location of lesions; their relationship with the biliary tree and vascular structures; and compression of adjacent organs. For example, in our case the cyst localized in the intrapericardial cavity may cause thickening of the pericardium, constrictive pericarditis, and/or cardiac tamponade. Anaphylactic reactions may also develop in some patients due to the antigenic properties of cystic fluid [8]. In our case, the patient had dyspnea and fatigue associated with cardiac compression due to pericardial effusion.

Transthoracic echocardiography, CT, and nuclear magnetic resonance imaging (MRI) have been successfully used in the diagnosis of cardiac hydatidosis. The diagnostic and/or therapeutic use of invasive techniques and interventional radiological procedures carries the risk of puncturing cysts, and this could lead to anaphylactic reaction, formation of secondary cysts, or even sudden death $[6,8]$.

Though successful results have been reported with mebendazole and albendazole, surgical therapy is still the favourite method for treating hydatid cysts. Some authors have suggested using albendazole as a supportive therapy for surgery in order to decrease the incidence of recurrence [6]. In this case, with the diagnosis of pericardial effusion and hepatic hydatid cyst, it was decided that surgery was the best treatment option associated with a complement of medical therapy.

\section{Conclusion}

Cardiac hydatid cysts should always be considered in the differential diagnosis of pericarditis or pericardial effusion, especially in regions where hydatid disease is endemic.

\section{Disclosures}

No disclosures.

\section{Competing interests}

The authors have no competing interests to report.

\section{Funding}

This research did not receive any specific grant from funding agencies in the public, commercial, or not-for-profit sectors.

\section{References}

1. Pakis I, Akyildiz EU, Karayel F, Turan AA, Senel B, et al. (2006) Sudden death due to an unrecognized cardiac hydatid cyst: three medicolegal autopsy cases. J Forensic Sci 51: 400-402. [Crossref]

2. Pedrosa I, Saiz A, Arrazola J, Ferreirós J, Pedrosa CS, et al. (2000) Hydatid Disease: Radiologic and Pathologic Features and Complications. Radiographics 20: 795-817. [Crossref]

3. Polat P, Kantarci M, Alper F, Suma S, Koruyucu MB, et al. (2003) Hydatid disease from head to toe. Radiographics 23: 475-494. [Crossref]

4. Dighiero J, Canabal EJ, Aguirre CV, Hazan J, Horjales JO (1958) Echinococcus disease of the heart. Circulation 17: 127-132.

5. Çakmak E, Alagozlu H, Gumus C, Alí C (2013) A case of Budd-Chiari syndrome associated with alveolar echinococcosis. J Pak Med Assoc 51: 475. [Crossref]

6. Mandolkar, SD, Ramakanth B, Sudarshan, GT (2016). Cystocutaneous fistula of the left lobe of liver: an extremely rare presentation of hydatid liver cyst. Int Surg J 2: 109-111.

7. Akay S, Erkan N, Yildirim M, Akay H (2015) Development of a cutaneous fistula following hepatic cystic echinococcosis. Springerplus 4: 538. [Crossref]

8. Ameli M, Mobarhan HA, Nouraii SS (1989) Surgical treatment of hydatid cysts of the heart: report of six cases. J Thorac Cardiovasc Surg 98: 892-901 [Crossref]

Copyright: (C2019 El Fihri LC. This is an open-access article distributed under the terms of the Creative Commons Attribution License, which permits unrestricted use, distribution, and reproduction in any medium, provided the original author and source are credited. 